\title{
Umwandlung von Cysteinylserin in Lanthionin
}

Im Verlauf unserer Arbeiten über den Schutz von Aminosäure-Seitenketten bei Peptidsynthesen sind wir folgendem Fall begegnet.

Bei der Umesterung von N-Benzyloxycarbonyl-Sbenzoyl-L-cysteinyl-L-serinmethylester (I, Smp. 174', $[\alpha]_{D}^{31}=-36.4^{\circ}$ in Dimethylformamid-DMF) mit methanolischer Natriummethylatlösung bildet sich erwartungsgemäss ${ }^{1}$ der S-benzoyl-freie Ester II; wird dagegen der entsprechende S, $O$-Dibenzoylester III (Smp. $147^{\circ},[\alpha]_{\mathrm{D}}^{31}=$
Wolle $^{3}$ etc. und von Insulin ${ }^{4}$, sowie an die Synthese von Lanthionin durch Wechselwirkung von Cystein und Acetylaminoacrylsäure in stark alkalischer Lösung ${ }^{5}$. Schliesslich möchten wir darauf hinweisen, dass Cyclolanthionylpeptide ein integrierender Bestandteil von Naturprodukten, z.B. Nisin, bilden ${ }^{6}$.

Nachtrag bei der Korrektur: die Substanz von Smp. 284-285 hat sich inzwischen als ein dimeres von $\mathrm{Vb}$ mit einem 14gliedrigen Ringsystem erwiesen.

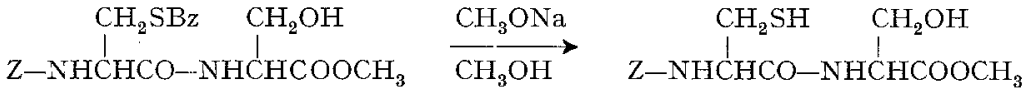

I

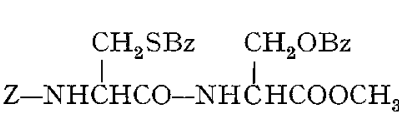
III

$$
\underset{\mathrm{CH}_{3} \mathrm{OH}}{\stackrel{\mathrm{CH}_{3} \mathrm{ONa}}{\longrightarrow}}
$$<smiles>[Z1]N[C@@H](CS)C(=O)N[C@H](C)C(=O)OC</smiles>
IV<smiles>COCCCCO</smiles><smiles>C1CCCC1</smiles><smiles>[Y]N[C@@H](C[PbH])C(=O)N[C@H](C[OH2+])C(=O)OC</smiles>
$\mathrm{VI}$

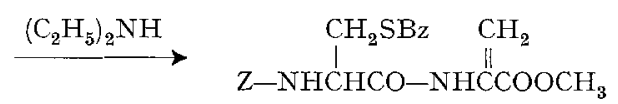

VII

$\mathrm{Z}=\mathrm{C}_{6} \mathrm{H}_{5} \mathrm{CH}_{2} \mathrm{OCO} ; \mathrm{Bz}=\mathrm{C}_{6} \mathrm{H}_{5} \mathrm{CO}-; \mathrm{Ts}=\mathrm{p}-\mathrm{CH}_{3} \mathrm{C}_{6} \mathrm{H}_{4} \mathrm{SO}_{2}$

$-41.3^{\circ}$ in DMF) auf dieselbe Weise behandelt, so wird der Serinanteil durch $\beta$-Abspaltung in Aminoacrylsäurederivat umgewandelt, während sich die gleichzeitig in Freiheit gesetzte SH-Gruppe an der entstandenen Doppelbindung addiert unter Bildung von N-Benzyloxycarbonylderivaten des L-Cyclolanthionylmethylesters (Va, Smp. $170^{\circ},[\alpha]_{\mathrm{D}}^{31}=+1.2^{\circ}$ in DMF) und des meso-Cyclolanthionylmethylesters (Vb, Smp. 284-285,$[\alpha]_{\mathrm{D}}^{31}=$ $-84.1^{\circ}$ in Dimethylsulfoxyd). Die beiden Stereoisomere $\mathrm{Va}$ und $\mathrm{Vb}$ entstehen auch, sogar in besserer Ausbeute, wenn man den aus dem S-Benzoyl-O-tosyldipeptidester VI (Smp. $120^{\circ},[\alpha]_{\mathrm{D}}^{31}=-37.8^{\circ}$ in DMF) in bekannter Weise ${ }^{2}$ durch $\beta$-Abspaltung erhältlichen N-BenzyloxycarbonylS-benzoyl-L-cysteinyl-aminoacrylsäuremethylester VII mit methanolischer Natriummethylatlösung behandelt. Anscheinend ist IV als Zwischenprodukt bei der Umwandlung von III $\rightarrow \mathrm{V}$ zu betrachten. Entcarbobenzoxylierung und anschliessende Hydrolyse von $\mathrm{Va}$ und $\mathrm{Vb}$ liefert L- bzw. meso-Lanthionin.

Der obige Reaktionsverlauf erinnert an die Bildung von Lanthionin bei der Hydrolyse von alkali-vorbehandelter
Summary. N-Benzyloxycarbonyl-S-benzoyl-L-cysteinyl$O$-benzoyl- $L$-serine methyl ester is transformed to $\mathrm{N}$ benzyloxycarbonyl derivatives of cyclo-L-lanthionyl methyl ester and of cyclo-meso-lanthionyl methyl ester.

\section{Zervas und N. Ferderigos}

Laboratorium für Organische Chemie,

$13 \mathrm{~A}$ Navarinou Street Universität Athen, Athen 144 (Griechenland), 14. August 1972

1 L. Zervas, I. Photaki und N. Ghelis, J. Am. chem. Soc. 85, 1337 (1963).

2 I. Photaki, J. Am. chem. Soc. 85, 1123 (1963).

3 M. J. Horn, D. B. Jones und S. J. Ringed, J. biol. Chem. 138, 141 (1941).

4 V. du Vigneaud, G. B. Brown und R. W. Bosnes, J. biol. Chem. 741, 707 (1941).

5 A. Schöbert und A. Wagner, Z. physiol, Chem. 304, 97 (1956).

6 E. Gross und J. L. Moreld, J. Am, chem. Soc. 93, 4634 (1971).

\section{The Dye Sensitized Photooxygenation of Kryptopyrrole}

In connection with chemical investigations into an increasingly widely applied phototherapy for neonatal jaundice ${ }^{1,2}$ there has been a renewed interest in the behavior of monopyrroles during photosensitized oxygenation $^{3}$. One of the most easily synthesized ${ }^{4}$ and biologically important monopyrroles ${ }^{5}$, kryptopyrrole (1), has been periodically examined following auto-oxidation ${ }^{6,7}$, after which the principal isolated products were found to have dimeric structures ${ }^{7}$. However, there have been no reports on the photooxygenation of 1. Because of their possible relationship to or existence as biological metabolites and their structural novelty, we wish to report on the photooxidation products of $\mathbf{1}$.

The photooxidation reaction was conducted in a water-cooled immersion apparatus containing a dilute $(0.84$ mmole \%) methanolic solution of kryptopyrrole (1) 